\title{
Developing the Concepts of Homeostasis, Homeorhesis, Allostasis, Elasticity, Flexibility and Plasticity of Brain Function
}

\author{
Alfredo Pereira, Jr.
}

Citation: Pereira, A., Jr. Developing the Concepts of Homeostasis,

Homeorhesis, Allostasis, Elasticity, Flexibility and Plasticity of Brain Function. NeuroSci 2021, 2, 372-382. https://doi.org/10.3390/

neurosci2040027

Academic Editor: Ricardo J. Rodrigues

Received: 22 September 2021

Accepted: 26 October 2021

Published: 5 November 2021

Publisher's Note: MDPI stays neutral with regard to jurisdictional claims in published maps and institutional affiliations.

Copyright: (C) 2021 by the author. Licensee MDPI, Basel, Switzerland. This article is an open access article distributed under the terms and conditions of the Creative Commons Attribution (CC BY) license (https:// creativecommons.org/licenses/by/ $4.0 /)$.
Institute of Biosciences, São Paulo State University (UNESP), Botucatu 18618-689, Brazil; alfredo.pereira@unesp.br
Abstract: I discuss some concepts advanced for the understanding of the complex dynamics of brain functions, and relate them to approaches in affective, cognitive and action neurosciences. These functions involve neuro-glial interactions in a dynamic system that receives sensory signals from the outside of the central nervous system, processes information in frequency, amplitude and phase-modulated electrochemical waves, and control muscles and glands to generate behavioral patterns. The astrocyte network is in charge of controlling global electrochemical homeostasis, and Hodgkin-Huxley dynamics drive the bioelectric homeostasis of single neurons. In elastic processes, perturbations cause instability, but the system returns to the basal equilibrium. In allostatic processes, perturbations elicit a response from the system, reacting to the deviation and driving the system to stable states far from the homeostatic equilibrium. When the system does not return to a fixed point or region of the state space, the process is called homeorhetic, and may present two types of evolution: (a) In flexible processes, there are previously existing "attractor" stable states that may be achieved after the perturbation, depending on context; (b) In plastic processes, the homeostatic set point(s) is(are) changed; the system is in a process of adaptation, in which the allostatic forces do not drive it back to the previous set point, but project to the new one. In the temporal phase from the deviant state to the recovery of stability, the system generates sensations that indicate if the recovery is successful (pleasure-like sensations) or if there is a failure (pain-like sensations).

Keywords: homeostasis; homeorhesis; allostasis; elasticity; flexibility; plasticity; brain function; orienting response

\section{Introduction}

Investigation of the local and global temporal dynamics of neural tissue is necessary for scientific and technological approaches to brain function. Understanding the role of the central nervous system (CNS) in perception-action cycles, generating conscious sensations (named qualia in the psycho-physical and philosophical literature) is also necessary for the study of behavior, involving the whole living body in the interaction with the changing physical and social environment.

The current state of investigation of temporal brain dynamics benefits from the discovery of astrocytes as being responsible for the control of brain global homeostasis [1]. In the human brain, external signals captured by sensors are sent to neuronal sensory networks and processed feed-forward to other neuronal systems, reaching parietal, temporal, and frontal 'associative areas' and the motor system, which initiates voluntary actions. A large number of synapses are tripartite [2], including one astrocyte together with two communicating neurons. The transmitters released by the pre-synaptic neuron also reach the astrocyte, binding with metabotropic receptors, activating signaling pathways that induce the formation of calcium waves $[3,4]$ by releasing the ions from the endoplasmic reticulum and propagation through gap junctions gated by connexins [5].

Besides this interaction with the environment, the SNC also interacts with the whole body in interoceptive cycles [6] mediated by neural and blood signaling, providing the SNC with 'somatic markers' [7]. The combination of perception-action with proprioceptive 
cycles generates temporal processes with different phases, depending on the epigenetic history of the living individual, contextual initial and boundary conditions.

Homeostasis, in this context, refers to a basal "set point" or equilibrium class of states of the CNS that compose, in elastic systems, the initial and putative target of the temporal process. Within the process, there are other phases to be considered; the phase corresponding to the deviation from equilibrium, and the phase of return to equilibrium, when an "orienting" response [8] is generated by the system, corresponding to a sensation [9] that helps to guide the process of homeostasis recovery.

There are conceptual and semantic issues in the neurosciences regarding the understanding of the "quality" of the orienting response. This response is a subjective experience of the system that is not accessible to the external, scientific observer. Besides many philosophical attempts to understand qualia, here, I put the emphasis on the efforts of neuroscientists such as Walter Freeman [10], Jaak Panksepp [11] and Antonio Damásio [12] to approach this issue, by means of concepts such as "meaning", "affect" and "feeling", respectively. In this paper, instead of a speculative view, I relate this type of subjective experience with a phase in the temporal dynamics of the CNS.

The astrocyte network controls global brain electrochemical homeostasis [1], while the Hodgkin-Huxley dynamics of depolarization and repolarization drives the bioelectric homeostasis of single neurons generating action potentials at the axon hillock. In complex systems as the brain, there may exist several stable regions of the state space ('attractors'), in which the system may rest far from the homeostatic equilibrium region. In this type of situation, we refer to a flexible system, instead of the elastic one. Furthermore, the system shaped by a series of changes affecting the molecular structure and functions and instantiating new stable regions is a plastic one. During development, there are changes in the state space of the system [13], implying changes in the distribution of set points; these changes are often labeled as "neural plasticity" [14]. In terms of dynamical systems theory, there is a change in the class of 'attractor regions' [15] of the state space, forcing the system to become adapted to new patterns of stability.

The theoretical framework I propose here is that the physiological dynamics of neural systems can basically be homeostatic (attached to an equilibrium point or region), or homeorhetic [16], when the dynamics drive the system away from the equilibrium point or region. Allostasis, in this context, refers to types of processes when homeorhesis leads to a new stable region of the system's state space. The temporal dynamics of the CNS after a perturbation may be elastic, when the process is strictly homeostatic; or flexible and/or plastic, when the process is allostatic. In the cases of flexible systems, the new stable region that is reached is a previously existing one that was kept "recessive". In the case of plastic systems, a new stable region is built by the process of interaction of the system with the whole body and environment.

\section{Signals, Homeostasis and Sensations: Orienting Reflex and Projective Models}

Signal processes in the CNS may be generated internally or externally of neural tissues. Each signal elicits a response from the tissue, which can be bioelectrical (ionic waves, spikes), chemical (release to transmitters, hormones) or molecular (activation of signal-transduction pathways by means of electrostatic protein conformation changes). The incoming signal perturbs the previous basal homeostatic state, causing a response that tends to drive the system back to homeostasis. This response and the resulting process back to stability are based on signaling processes and physiological responses.

In this temporal process, "differences make differences". The first differences are differences within the signals (non-redundancy), and the second difference is the deviation from homeostasis caused by the signal. The sentient [9] capacity of neural systems depends on the process during which sensations emerge, corresponding to a phase in the process of allostasis. A perturbation (difference in stimulation) causes a deviation from homeostasis (difference in physiological activity), to which the system reacts by returning to the homeostatic basal state. At this moment, sensations are experienced together with the effort 
to return to homeostasis. If the system is successful (e.g., drinking water to satiety), then sensations are "positive" (pleasure-like); but if the system fails (e.g., continued lesion of tissue) then sensations are "negative" (pain-like).

A crucial step in understanding the dynamics of allostasis and generation of sensations was initially given by Sokolov's observation that the presentation of a habitual stimulus does not elicit a recognition process, but the withdrawal of such a stimulus does. As Martindale describes, "According to Sokolov, the cortex compares all incoming stimuli with models or expectations. If the incoming stimulus matches a pre-existing cortical model, the cortex blocks the reticular system and ... no attention is paid to the stimulus. On the other hand, if the stimulus is novel - that is, if it does not exactly match any cortical model-then the cortex does not block the reticular system" [17].

The withdrawal of a previously habitual stimulus constitutes in itself a new stimulus, to which attention is directed. The whole dynamics of recognition processes can be summarized in Sokolov's match-mismatch rule: "If a percept exactly matches a set of cognitive units, nothing happens other than passive perception. If a percept matches the best-fitting set of cognitive units, then the arousal system activates the cortex. This arousal response is accompanied by an orienting reflex, attention, and the construction of a new cortical model" [17].

E. Sokolov and O. Vinogradova [8] related the orienting reflex with the functions carried by the limbic system. In this expression, the word "reflex" does not mean a response elicited by an external conditioning; on the contrary, it refers to a constructive response (based on unconditioned affective dispositions) that guides behavior, in the following kinds of situations:

(a) When a stereotyped pattern is initiated, without being triggered by a specific stimulus, e.g., a chicken searching for food, in the absence of any specific food stimulus.

(b) When a stereotyped pattern is triggered by a specific stimulus, e.g., a predator-like sound inducing the prey to escape.

(c) When there is a stereotyped reaction to a change in a habitual environmental setting, e.g., the miller who wakes up when the noisy mill stops working.

One role of the limbic system in rodents would be signaling changes among types of behavior. Sokolov and associates tested this hypothesis experimentally, at the neuronal level, focusing on cases B and C above ([8], pp. 226, 233).

An analysis of how habituation works at the ontogenetic scale [18] suggests a possible phylogenetic adaptation for the recognition of a distal stimulus from proximal signals. For example, when we hold a tool with the hand, e.g., a hammer, we first experience touch with the tool, but as we use it we become habituated and the focus of attention turns to the interface between the hammer and the part of the environment that offers a resistance to it, e.g., the nail.

The signals generated internally to the brain are the most proximal stimulus, the ones to which we are phylogenetically most habituated. Is it possible that in the distant evolutionary past, or in some artificial situation, the brain could perceive its own workings? It is possible to amplify a signal from an electrode placed in the scalp, making it possible for the brain to monitor its own activities, a therapeutic practice called "biofeedback" $[19,20]$. Another example is closing the eyes and pressing the eyeballs with a finger; some phosphorous phantoms can usually be seen, presumably produced by remaining excitatory activity of retinal cells. In both examples, the nervous system perceives a fragment of its own workings, but in normal adaptive situations ancient habituation mechanisms preclude literal brain self-reference. These examples serve to demonstrate that it is not impossible for the brain to perceive parts of itself, hence suggesting that projective intentionality would be generated by habituation mechanisms that block the respective sensation.

Another precursor of this view is Freud. His "Project for a Scientific Psychology" refers to a "dissipation" process that corresponds to our conjecture of allostatic recovery of stability: "Freud defined this as the tendency of neurons to divest themselves completely of the quantities of excitation (endogenous or exogenous) that erupt into the psychical 
apparatus. The primary function of this apparatus would therefore be to reduce to the lowest level possible-ideally a 'level = zero' - the quantity of free energy" [21].

The initial contributions of the Sokolov group have been resumed in current approaches to the projective capabilities of the brain. The Freudian hypothesis, later refined in the concepts of a principle of pleasure and the death drive, has recently been put into perspective in the neuroscientific work of Karl Friston and his colleagues with the Bayesian approach, "Its aim being to avoid too great a variation in the quantity of free energy coming from our sensorial perceptions (both internal and external) on the basis of prediction of sensorial data. This offers an unexpected point of dialog between psychoanalysis and neuroscience centered on an energetics concept of cerebral function." [21].

The generation of sensations is not explained by homeostasis (the forces that drive the system to stay at the set point) alone, but require reference to another explanatory factor: an "elastic" property that drives the system move to another stable state once a perturbation (stimulus) moved it to a distance from the equilibrium. For instance, in sexual experiences the differences in the stimulation cause a difference in the homeostasis (deviation from the equilibrium) and then, after an excitation threshold is crossed, we feel pleasure (orgasm) as a signal of the return to homeostatic equilibrium. At the homeostatic equilibrium we do not feel pleasure or any other sensation. Therefore, sensations are dynamic temporal patterns experienced in the process of homeostasis deviation and allostatic recovery. This process involves a projective operation in which conscious sensations are generated and attributed to a source of stimulation that is often located externally to the brain [22]. We feel the pain of a tissue lesion in the foot as being located in the foot, not in the brain. Scientifically we know that the sensation of pain is produced in the brain, because the blockage of the signal from the foot to the brain by a local anesthetic abolishes the sensation.

In this approach, sensations have an adaptive function, both internally-to participate in the mechanism of control of brain homeostasis-and externally-to react correctly to the environmental stimulus. For instance, when we feel pain after sticking a finger in a rose thorn, we withdraw the finger and then the homeostasis of the skin tissue is recovered. There are also endocrine, immune and autonomic actions on the physiology (the "psychosomatic effect") by means of hormones, peptides, modulators, antibodies and other signaling molecules, which can-in flexible systems—change the homeostatic set point and then help us to live with the bad stimulus without becoming severely ill or feeling pain.

In plastic systems, such as brains, the continued changes in homeostatic set points cause irreversible and permanent changes in the functioning of neural tissues, including genetic regulation and differential expression of signals that control the growth of dendrites and axons. This type of process has been proved to be essential to the process of learning and formation of long-term memory [23].

\section{Glutamate and Calcium Mechanisms in the Control of Basal Homeostasis}

The basal mechanism of homeostasis in the brain is composed of excitatory and inhibitory balancing factors and has a fixed equilibrium set point. Glutamate (Glu) is considered to be the main excitatory neurotransmitter in the brain, present in corticocortical networks and binding to receptors of both excitatory (as pyramidal cortical) and inhibitory neurons (as GABA interneurons). The excitation of interneurons increases their inhibition (i.e., GABAergic transmission) of other neurons. Therefore, this is a central component in the balance of excitation and inhibition, and the balance, in turn, is a condition for the operation of information transmission networks that support several brain functions. The temporal process of excitation and inhibition provides the brain with a dynamic mechanism necessary for adequate responses to environmental stimuli and respective adaptive actions.

Thalamo-cortical and cortico-cortical synapses are central for the understanding of perceptual processing in the brain. Information from the environment is carried in time by neuronal action potentials, forming spike trains, which are transduced at each synapse, reaching the sensory cortex and associative areas. Once reaching these areas, the excitatory 
input activates specialized feature detectors. The central nervous system (CNS) constructs integrated scenes, using ensembles of feature detectors, spatio-temporally activated by the information patterns carried by the series of neurons involved in the input processing.

In the mammalian brain, the excitatory sensory signals initially pass (except olfactory signals) through thalamic relay cells and reach the fourth layer of a cortical column. According to a simplified model [24], neurons composing the latter preferentially fire to other neurons located in the fifth and sixth layers of the same column. After being excited, these neurons fire back to the thalamus (operating as a confirmation signal) and also send the excitatory signal to superficial layers of the cortex, where the corresponding neurons activate cortico-cortical connections, horizontally spreading the excitation to other cortical columns. After a temporal interval, relative to the content being processed, the excitatory process is extinguished by habituation mechanisms involving the excitation of thalamic and cortical inhibitory interneurons [24]. With this mechanism, the excitatory process dynamically moves through neocortical columns, putatively composing the "flux of thought".

The role of Glu as information carrier in thalamocortical and cortico-cortical synapses is crucial for the understanding of how perceptual learning is possible in the brain. Glu mediates the "flux of thought", and for this reason a hypothesis was raised that it should have a central role in the generation of conscious contents, even in dreams and altered states of consciousness. This hypothesis was tested in experiments when the Glu NMDA receptor is blocked by an antagonist (ketamine, PCP or MK-801), and the results indicated that the blocakage elicits perceptual distortions and hallucinations [25].

It is well known from EEG methods and recordings that spike trains encode information patterns by means of frequency and phase modulation in populations of axons. The CNS uses these patterns to construct consciously experienced episodes in temporal windows of approximately 2 to $3 \mathrm{~s}$ [26]. The concepts of feature-detectors and populationrate coding have been combined in the idea of a sparse population code [27]. The detection of real-world objects and processes and the construction of the corresponding conscious episodes involve cooperative groups of neurons, forming the so-called "Hebbian Assemblies", which are relatively small cell populations displaying strengthened connections built by previous learning [28].

The input patterns impacting on neuronal membrane receptors control intracellular signal transduction pathways that reach the dendritic spines. At these sites, there is a molecular processing of the relevant apatterns, supporting conscious perception, selective memory formation and unconscious priming. The mechanism involved in such a recording of sensory patterns has been studied as the early stage of LTP [29]. It involves biological molecular structures and functions, including the system of Glu receptors, and calciumbinding proteins such as calmodulin $(\mathrm{CaM})$ and calmodulin-dependent protein kinase II (CamKII, a protein from the kinase family, having several receptor and effector active sites) [24].

In the sensory cortex, input information patterns transmitted through thalamocortical glutamatergic projections are received and processed by post-synaptic mechanisms. Glu released from the pre-synaptic neuron binds to three kinds of receptors (AMPA, NMDA and metabotropic Glu receptors-MetGR) at the post-synaptic membrane. The three kinds of receptors activate signal-transduction pathways that converge into the dendritic spine [30], controlling CaM/CaMKII signaling.

Calcium cations $\left(\mathrm{Ca}^{++}\right)$are largely employed biological ions that encode information patterns. CaM and CamKII have several receptor and effector active sites for the $\mathrm{Ca}^{++}$ions entering through NMDA and VDCCs. The ions trapped in CaM are processed by the kinase, triggering regulatory functions. The informational state of CaM/CaMKII is dependent on the interaction with the $\mathrm{Ca}^{++}$population passing through NMDA and VDCCs. Most of $\mathrm{Ca}^{++}$entry is made through the NMDA channel, a "coincidence-detector" for sensory afferent and previously learned patterns [24,31]. The NMDA channel assures the reliability of perception in regard to stimuli, since it is opened only if endogenous and afferent pulses 
reach the NMDA subunits together. If VDCCs (which are not coincidence detectors) assume the main role in glutamatergic transmission, perceptual distortions and hallucinations may occur.

The complex structure of CamKII, having binding sites for $\mathrm{Ca}^{++}$and phosphatases that act on other proteins, constitutes a micro device able to read quantized information from $\mathrm{Ca}^{++}$, to process this information and activate other proteins. The multimeric structure of CaMKII contains four sites that bind to CaM, determining the conformational state of the kinase and the resulting phosphorylation functions [24]. $\mathrm{Ca}^{++}$-activated CaM can trigger several signaling pathways in the cell, some of them exerting feedback control on the state of the membrane, and others reaching the nucleus and inducing the formation of long-term memory.

The above signaling is related to mental processes that depend on the glutamatergic mechanisms responsible for perceptual learning. One of the proteins present in the converging Glu receptor pathways is DARPP-32, which is found in the striatum, controlling thalamocortical glutamatergic and cholinergic neurons with the participation of dopaminergic modulation [32]. Striatal signals processed by such converging molecular pathways convey information to the cortex as an "efferent copy" or "corollary discharge" (i.e., signals sent from motor to perceptual areas when a voluntary action is initiated). DARPP-32 activity, connecting dopaminergic and Glu-activated signal transduction pathways, has been related to schizophrenia, participating in its positive, negative and cognitive symptoms. An explanation for the role of DARPP-32, and for the cognitive deficits that occur when its respective signaling pathways are disturbed, is that the "efferent copy" would have the role of reinforcing perceived patterns, increasing their chance to form long-term memories.

\section{Synaptic Plasticity and Homeorhesis}

The instantiation of information patterns in neural tissue and computational processes using these patterns correspond to the process of sequential excitation and inhibition of cortical columns $[24,33,34]$. At the level of each column, the homeostatic set point is previously established by long-term memory macromolecular mechanisms that establish the "weight" or "strength" of connections that define the basal type of state (resting electrical potential). During the lifetime experience of an individual, changes in the production and release of neurotransmitters [35,36], resulting in differential concentrations at the synapse, can change the set point. These changes correspond to homeorhetic processes, moving the system beyond the simpler homeostasis of excitation and inhibition. Together with the homeorhetic process, the flexible or plastic reaction of the system to the changes in the set point can elicit a response that is experienced as a sensation or feeling of having learned something new and having knowledge of it. In the case of a complete process of learning, acquiring knowledge and becoming aware of it by means of the "feeling of knowing", the whole process can be considered allostatic.

The formation of cognitive contents ("representations") in conscious episodes comprises a deviation from the basal family of states and returning to the same type of state (homeostasis, in elastic processes) or reaching another stability region (homeorhesis, in flexible and plastic processes). A representation corresponds to a pattern of active connections [37] in neuronal networks, leading to a temporal pattern of neuron firing that communicates the result to other neurons in charge of controlling muscles and glands, thus generating behavioral patterns. These excitations are not static, but under a physiological dynamic process of habituation and response to novelties that perturb the system and drive it away from homeostatic equilibrium. Neural assemblies activated at one moment are inhibited in the next moment, while the chain of excitations move along neocortical tissues radially connected with the thalamus [34]. The temporal chain of thought corresponds to a series of excitations and inhibitions moving along neocortical tissue. The control is the response of the system (using Sokolov's terminology, it is the orientation of the system that supports the process of adaptation to the changing environment), directing the dynamics of connections back to the synaptic basal level of activity determined by long-term 
potentiation or to another stability region, by means of changes in the concentration of neuromodulators.

The dynamics of spiking neurons and their patterns of connectivity in cortical tissue have the characteristics of complex dynamical systems, in which the trajectory moves through a rich landscape containing many attractors (see $[38,39])$. Changes in axonal signaling (spike trains) and resulting changes in the strength or weight of synaptic connections can compose an allostatic process, because of flexible or plastic changes in neuromodulation [35], leading the system to occupy new regions of the state space and constructively building on the new experiences. After the allostatic process, synaptic homeostasis is guided by another set point or region in the system's state space. In experiential terms, this type of learning process is accompanied by the "feeling of knowing" something new. This feeling is, in the proposed framework, an experiential correlate of the cognitive allostatic process.

\section{Neuron-Astrocyte Interactions}

Most glutamatergic synapses are "tripartite" (in the sense of being composed of two neurons and one astrocyte cells). The Glu released by the presynaptic neuron reaches both the postsynaptic neuron and the neighbor astrocyte that wraps the synaptic space. Glu induces membrane depolarization, according to the previously cited mechanisms, prompting the opening of NMDA channels. The excitation decays in around $150 \mathrm{~ms}$ [24] but can be sustained by gliotransmission (by the Glu released by the astrocyte, which binds to extra NMDA receptors), producing slow calcium currents, which in turn activates the path of calmodulin and its kinase (CaMKII). By phosphorylating or dephosphorylating AMPA receptors, the processes initiated by glial Glu can induce both synaptic potentiation and depression.

When the animal is becoming awake, the astrocytic network is pre-activated by adrenergic, purinergic and cholinergic mechanisms, facilitating the generation of calcium waves at the time when Glu transmission occurs [24]. In the astrocyte, the induction of local calcium waves by means of excitatory transmission generates intercellular, global waves that broadcast information to many other parts of the brain $[3,4]$.

While in the "Neuron Doctrine" (formulated by Ramon y Cajal) neurons were considered the functional units of the mind, in the new emerging model of neuro-astroglial interactions the tripartite synapse becomes the functional unit, constituting the basis for psycho-physiological processes. Neural networks are mostly responsible for cognitive processes (such as the formation of representations and logical operations in processes of perception, attention, action, learning and memory formation), whereas the astrocyte network performs an appreciation of the information carried by the neural network, putatively by means of intercellular, global calcium waves. Based on the appreciation, the astrocyte network modulates neuronal activity, reinforcing the patterns of information that have positive valence for the individual, and weakening those with negative valence [24]. These complex neuron-astrocyte interactions can elicit flexible and plastic processes mediated by signals between the two types of cell populations [3]. From neurons to astrocytes, several transmitters can be used, while from astrocytes to neurons-besides the release of gliotransmitters by means of transporters and vesicles-experimental research has revealed a central role of potassium ion concentrations controlled by the astrocyte [40].

The functions of integration and appreciation of information can be considered as closely related, because any lived experience intrinsically contains an appraisal, assigning positive, neutral or negative valence to aspects of the experience. The astroglial network participates in both processes of integration and appreciation of information patterns, which are previously processed in a distributed manner in the thalamocortical system, interacting with somatic processes by means of signaling via blood flow and cerebral fluid. The astroglial network putatively makes the appraisal to aptly modulate the neuronal network, participating in processes of perception, attention, learning, training of semantic and episodic memory, emotion, consciousness and control of behavior $[3,4,24]$. 
As the astrocytes are in contact with blood and cerebrospinal fluid, by means of communicating channels present in their processes, they receive signals that come in the flow, allowing a continuously updated appraisal of the state of the body. The intracellular astrocyte signaling generates small calcium waves that interfere with each other, resulting in larger waves built by means of constructive interference [24]. The intrinsic patterns of the larger waves, to be studied experimentally, were proposed to correspond to the first-person experienced feelings [4].

Astrocytes are not connected to sensory transducers and muscle or endocrine effectors; sensation and perception, as well as actions in the world, begin with neurons. Information patterns embodied in local electromagnetic fields of neurons reach the astrocytes and induce the larger waves (see the 'domino' and 'carousel' effects [3]). The larger waves in the astrocyte network require a coordinating action from neurons [24]:

(a) Local EM fields are formed by active neuronal assemblies.

(b) Neuronal large-scale synchronization occurs in theta to gamma frequencies (not delta; synchronization in the slowest frequencies imply unconsciousness).

(c) There are chemical and ephaptic (magnetic) transmissions of information from the neuronal local fields to astroglial waves; and

(d) There is an interference of the smaller waves, leading to the formation of the larger ones in the astroglial network.

\section{The Hydro-Ionic Wave and Allostasis}

The neuro-astroglial interaction model has theoretical resources to address not only the cognitive representation of emotions, but also the lived experience of conscious sensations by whole biological individuals. Amplitude-modulated ionic waves in astrocytes and extracellular fluid are regarded as the carriers of the sensory affective or emotional response to a perturbation of homeostasis. The elicited process may be allostatic, leading the system to stable states far from homeostatic equilibrium. In this regard, the "Endogenous Feedback Network" hypothesis [41] advanced the idea that conscious feeling involves the interaction of neuronal and astroglial networks. The first may be considered responsible for the processing of afferent and efferent information patterns and the constructing of representations, while the second would be responsible for the direct experience of feeling.

The concept of allostasis, in this context, is co-extensive to learning and memory [42] processes at cellular and tissue scales, as treated in computational modelling. In neurobiological language, authors refer to processes of reciprocal modulation [3,43], regulation [44] and control [45] of neurons and astrocytes, generating as a result the phenomenon of neural plasticity [14,46]. This process is more intense during the development stages [47] and persists during the whole life of biological individuals, determining processes of health and disease [48]. The connection with cognitive learning, i.e., the formation of new memories, can be exemplified by neurophysiological studies of neuron-astrocyte interactions in the hippocampus $[49,50]$.

The temporal process in which neurons activate calcium waves in astrocytes and these waves modulate neurons in the following time interval is the following (see also the "carousel effect" concept advanced in [3]): A given neural microstate generates both the EEG signal and changes in the previously existing standing calcium wave in astrocytes, by means of the release of transmitters [43]. In the next moment, the calcium wave modulates the following microstate, reinforcing patterns with systemic positive valence, and depressing patterns with systemic negative valence. The second microstate generates both the next EEG register and the changes in the existing calcium wave, and so on. Although the scalp EEG does not measure the calcium wave directly, the modulatory function exerted by the wave is implicit in the temporal evolution of the registered electromagnetic signal [24].

\section{Summary of Concepts}

Based on the above considerations, I summarize the concepts of homeostasis, homeorhesis, allostasis, elasticity, flexibility and plasticity used in this paper: 
(a) Homeostasis: A physiological (temporal) process in which the system has a fixed point or region of equilibrium, and returns to this point or region after a perturbation.

(b) Homeorhesis: A physiological (temporal) process in which perturbations destroy the system's previous fixed point or region and drives it to an unstable region of the state space.

(c) Allostasis: A physiological (temporal) process in which the system moves away from the basal equilibrium point or region, crosses an unstable phase and then achieves a new stable point or region, driven by an adaptive response based on tissue waves that may be related to conscious sensations.

(d) Elasticity: A property of homeostatic systems returning to equilibrium after a perturbation.

(e) Flexibility: A property of homeorhetic systems responding to perturbations alternating between equilibrium points or regions. It implies a diversity of attractors.

(f) Plasticity: A property of allostatic systems creating new attractors far from equilibrium, on the basis of internal and external (adaptive) interactions. The creation of new attractors implies a psycho-physical learning process and formation of long-term memory and, therefore, a degree of cognitive and enactive processing together with the experiencing of basic sensations.

\section{Concluding Remarks}

The dynamics of the generation of sensations in neural tissues, in elastic, flexible and plastic systems, can be illustrated by the exchange of potential and kinetic energy in the movement of a pendulum. At one point there is only potential energy, and at another point there is only kinetic energy; between them, there are all possible combinations of both, on a continuum. The same reasoning applies to neural systems. During slow-wave sleep, there are only potential sensations and during wakefulness there are moments when we are making the most of our potentialities [34].

The circadian rhythm in chronobiology concerns these oscillations experienced across the 24-h period. In addition, there are faster oscillations that happen at every moment, and several combinations of potential and actual consciousness, from dreaming (in which a maximum of conscious control in realized in lucid dreaming) to varied experiences in wakefulness, from empty minds to mindfulness. The brain process is continuous, but there are three relevant differences between the dynamics of the pendulum analogy and the functioning of neural tissues [34]:

(a) The existence of a 'switch' between unconscious sleep and wakefulness, but not in the movement of the pendulum. However, the existence of a 'switch' does not mean that non-consciousness and consciousness compose a binary structure. The 'switch' is a point on a continuum, such as the transition from liquid to gaseous water at 100 degrees centigrade; although there is a phase transition, it does not imply a discontinuity in the temporal process. For the first-person perspective of the biological individual, sensations are felt only when the system crosses this threshold.

(b) The "state of pure potentiality" of the pendulum is just a point on a continuum, whereas this point practically does not exist for conscious living systems; it exists only for our corpses when we are completely dead, because even when we are alive and not conscious there is some degree of basal activity.

(c) A fixed point of equilibrium exists only in artificial devices such as thermostats, not in living systems. Indeed, even for a perfect gas in a closed container, the equilibrium modes (the 'Maxwellian equilibrium') are active, consisting, as they do, of Brownian motion. The conscious system is always active, therefore the basal/equilibrium state is a class of states changing within a region of the state space of the system. A stimulus impinging on the system changes the regimen or mode of activity, but the system is already active.

In sum, the dynamic physiological processes that generate sensations and feelings are composed of three phases [34]: 
(1) A dynamical baseline that embodies the structural potentialities of the system. The structure and the derived dynamics, together with the existing boundary conditions, determine if the system is sentient (capable of feeling) or not. A thermostat is not sentient because the equilibrium is defined-by the engineer who designed it—as a set point.

(2) An unstable phase in which a stimulus (internal or external to the system) drives the trajectory away from homeostatic equilibrium and generates a response (the hydro-ionic wave) that supports a qualitative experience (sensation) in the firstperson perspective of the system. If the process moves to an adaptive resolution, the experienced sensation is more likely to be pleasant; if not, it is likely to be unpleasant.

(3) The recovery of stability, when the system returns to the homeostatic equilibrium class of states (elastic systems) or moves to another class of states (attractor region of the state space, in flexible and plastic systems).

To account for both the physiological processes discussed in this paper, and the psychological processes experienced by the individual, I have proposed [34] the distinction of sentiomics (objective study of dynamical patterns involved in the generation of sensations, feelings and emotions, in the third-person perspective) and qualiomics (the study of feeling experiences in the first-person perspective).

Funding: This research received no external funding.

Conflicts of Interest: The author declares no conflict of interest.

\section{References}

1. Verkhratsky, A.; Nedergaard, M. Physiology of astroglia. Physiol. Rev. 2018, 98, 239-389. [CrossRef]

2. Pereira, A., Jr.; Furlan, F.A. Analog modeling of human cognitive functions with tripartite synapses. In Model-Based Reasoning in Science and Technology; Springer: Berlin/Heidelberg, Germany, 2011; Volume 314, pp. 623-635.

3. Pereira, A., Jr.; Furlan, F.A. Astrocytes and human cognition: Modeling information integration and modulation of neuronal activity. Prog. Neurobiol. 2010, 92, 405-420. [CrossRef] [PubMed]

4. Pereira, A., Jr. Astroglial hydro-ionic waves guided by the extracellular matrix: An exploratory model. J. Integr. Neurosci. 2017, 16, 57-72. [CrossRef] [PubMed]

5. Robertson, J.M. Astrocyte domains and the three-dimensional and seamless expression of consciousness and explicit memories. Med. Hypotheses 2016, 81, 1017-1024. [CrossRef] [PubMed]

6. Critchley, H.D.; Garfinkel, S.N. Interoception and emotion. Curr. Opin. Psychol. 2017, 17, 7-14. [CrossRef]

7. Damásio, A. Descartes' Error: Emotion, Reason, and the Human Brain; Grosset/Putnam: New York, NY, USA, 1994.

8. Sokolov, E.N.; Vinogradova, O.S. The Neuronal Mechanisms of the Orienting Reflex; Laurence Erlbaum Associates Publishers: Hillsdale, MI, USA; New York, NY, USA, 1975.

9. Pereira, A., Jr. The role of sentience in the theory of consciousness and medical practice. J. Conscious. Stud. 2021, 28, 22-50.

10. Freeman, W.J. Societies of Brains; Lawrence Erlbaum: Hillsdale, MI, USA, 1995.

11. Panksepp, J. Affective Neuroscience: The Foundations of Human and Animal Emotions; Oxford University Press: New York, NY, USA, 1998.

12. Damásio, A. The Feeling of What Happens: Body and Emotion in the Making of Consciousness; Harcourt: New York, NY, USA, 1999.

13. Fell, J. Identifying neural correlates of consciousness: The state space approach. Conscious. Cogn. 2004, 13, 709-729. [CrossRef] [PubMed]

14. Wang, F.; Yuan, T.; Pereira, A., Jr;; Verkhratsky, A.; Huang, J.H. Glial cells and synaptic plasticity. Neural Plast. 2016, 2016, 5042902. [CrossRef]

15. Schöner, G.; Zanone, P.G.; Kelso, J.A. Learning as change of coordination dynamics: Theory and experiment. J. Mot. Behav. 1992, 24, 29-48. [CrossRef]

16. Pace-Schott, E.F.; Amole, M.C.; Aue, T.; Balconi, M.; Bylsma, L.M.; Critchley, H.; Demaree, H.A.; Friedman, B.H.; Gooding, A.E.K.; Gosseries, O.; et al. Physiological feelings. Neurosci. Biobehav. Rev. 2019, 103, 267-304. [CrossRef]

17. Martindale, C. Cognition and Consciousness; Dorsey: Homewood, IL, USA, 1981.

18. Gray, J.A. The contents of consciousness: A neuropsychological conjecture. Behav. Brain Sci. 1995, 18, 659-722. [CrossRef]

19. Sterman, M.B.; Macdonald, L. Effects of central cortical eeg feedback training on incidence of poorly controlled seizures. Epilepsia 1978, 19, 207-222. [CrossRef]

20. Hauri, P.J.; Percy, L.; Hellekson, C.; Hartman, E.; Russ, D. The treatment of psychophysiologic insomnia with biofeedback: A replication study. Biofeedback Self-Regul. 1982, 7, 223-236. [CrossRef]

21. Tran, J.; Magistretti, P.; Ansermet, F. The epistemological foundations of freud's energetics model. Front. Psychol. 2018, 9, 1861. [CrossRef]

22. Pereira, A., Jr. The projective theory of consciousness: From neuroscience to philosophical psychology. Trans/Form/Ação 2018, 41, 199-232. [CrossRef]

23. Bailey, C.H.; Kandel, E.R. Molecular and structural mechanisms underlying long-term memory. In The Cognitive Neurosciences; Gazzaniga, M., Ed.; MIT Press: Cambridge, MA, USA, 1995. 
24. Pereira, A., Jr. Classical-quantum interfaces in living neural tissue supporting conscious functions. In Advances in Quantum Chemistry 82; Poznanski, R., Brändas, E., Eds.; Elsevier: Amsterdam, The Netherlands, 2020.

25. Pereira, A., Jr.; Johnson, G. Towards an understanding of the genesis of ketamine-induced perceptual distortions and hallucinations. Brain Mind 2003, 4, 307-326. [CrossRef]

26. Pereira, A., Jr.; Foz, F.B.; Rocha, A.F. The dynamical signature of conscious processing: From modality-specific percepts to complex episodes. Psychol. Conscious. Theory Res. Pract. 2017, 4, 230-247. [CrossRef]

27. Fotheringhame, D.K.; Young, M.P. Neural coding schemes for sensory representation: Theoretical proposals and empirical evidence. In Cognitive Neuroscience; Rugg, M.D., Ed.; MIT Press: Cambridge, MA, USA, 1997.

28. Phillips, W.A. Theories of cortical computation. In Cognitive Neuroscience; Rugg, M.A., Ed.; MIT Press: Cambridge, MA, USA, 1997.

29. Bickle, J. Philosophy and Neuroscience: A Ruthless Reductive Account; Kluwer: Dordrecht, The Netherlands, 2003.

30. Sabatini, B.L.; Maravall, M.; Svoboda, K. Ca ${ }^{2+}$ signaling in dendritic spines. Curr. Opin. Neurobiol. 2001, 11, 349-356. [CrossRef]

31. Rocha, A.F.; Pereira, J.R.; Coutinho, F.A.B. N-methyl-D-aspartate channel and consciousness: From signal coincidence detection to quantum computing. Prog. Neurobiol. 2001, 64, 555-573. [CrossRef]

32. Svenningsson, P.; Tzavara, E.T.; Carruthers, R.; Rachleff, I.; Wattler, S.; Nehls, M.; Mckinzie, D.L.; Fienberg, A.A.; Nomikos, G.G.; Greengard, P. Diverse psychotomimetics act through a common signaling pathway. Science 2003, 302, 1412-1415. [CrossRef]

33. Pereira, A., Jr. Epistemological Issues in Cognitive Neuroscience; Scholars' Press: Riga, Latvia, 2018.

34. Pereira, A., Jr. Reply to commentaries and future directions. J. Conscious. Stud. 2021, 28, 199-228.

35. Wang, F.; Pereira, A., Jr. Neuromodulation, emotional feelings and affective disorders. Mens Sana Monogr. 2016, 14, 5-29.

36. Upreti, C.; Konstantinov, E.; Kassabov, S.R.; Bailey, C.H.; Kandel, E.R. Serotonin induces structural plasticity of both extrinsic modulating and intrinsic mediating circuits in vitro in aplysia californica. Cell Rep. 2019, 28, 2955-2965. [CrossRef]

37. Churchland, P. Neurophilosophy: Towards a Unified Approach of the Mind/Brain; MIT Press: Cambridge, MA, USA, 1986.

38. Pereira, A., Jr.; Vimal, R.L.P.; Pregnolato, M. Can qualitative physics solve the hard problem? In Biophysics of Consciousness: A Foundational Approach; Poznanski, R., Tuszynski, J., Feinberg, T., Eds.; World Scientific: Singapore, 2017.

39. Nunn, C. From Neurons to Notions: Brains, Mind and Meaning; Floris Books: Edinburgh, UK, 2008.

40. Beckner, M.E. A roadmap for potassium buffering/dispersion via the glial network of the CNS. Neurochem. Int. 2020, 136, 104727. [CrossRef]

41. Carrara-Augustenborg, C.; Pereira, A., Jr. Brain endogenous feedback and degrees of consciousness. In Consciousness: States, Mechanisms and Disorders; Cavanna, A.E., Nani, A., Eds.; Nova Science: New York, NY, USA, 2012.

42. Kozachkov, L.; Michmizos, K.P. Sequence learning in associative neuronal-astrocytic networks. In Brain Informatics 2020; Lecture Notes in Computer Science Book Series; Mahmud, M., Vassanelli, S., Kaiser, M.S., Zhong, N., Eds.; Springer: Cham, Switzerland, 2020; Volume 12241.

43. Wotton, C.A.; Cross, C.D.; Bekar, L.K. Serotonin, norepinephrine, and acetylcholine differentially affect astrocytic potassium clearance to modulate somatosensory signaling in male mice. J. Neurosci. Res. 2020, 98, 964-977. [CrossRef]

44. Kottmeier, R.; Bittern, J.; Schoofs, A.; Scheiwe, F.; Matzat, T.; Pankratz, M.; Klämbt, C. Wrapping glia regulates neuronal signaling speed and precision in the peripheral nervous system of Drosophila. Nat. Commun. 2020, 11, 4491. [CrossRef] [PubMed]

45. Ryczko, D.; Hanini-Daoud, M.; Condamine, S.; Bréant, B.J.B.; Fougère, M.; Araya, R.; Kolta, A. S100 $\beta$-mediated astroglial control of firing and input processing in layer 5 pyramidal neurons of the mouse visual cortex. J. Physiol. 2021, 599, 677-707. [CrossRef]

46. Iannella, N.; Condemine, M. Neurons and plasticity: What do glial cells have to do with this? In Functional Brain Mapping: Methods and Aims; Tsytsarev, V., Yamamoto, V., Zhong, N., Eds.; Springer: Singapore, 2020.

47. Perez-Catalan, N.A.; Doe, C.Q.; Ackerman, S.D. The role of astrocyte-mediated plasticity in neural circuit development and function. Neural Dev. 2021, 16, 1-14. [CrossRef] [PubMed]

48. Pistono, C.; Bister, N.; Stanová, I.; Malm, T. Glia-derived extracellular vesicles: Role in central nervous system communication in health and disease. Front. Cell Dev. Biol. 2021, 8, 623771. [CrossRef]

49. Rose, C.R.; Felix, L.; Zeug, A.; Dietrich, D.; Reiner, A.; Henneberger, C. Astroglial glutamate signaling and uptake in the hippocampus. Front. Mol. Neurosci. 2018, 10, 451. [CrossRef]

50. Lebedeva, A.; Plata, A.; Nosova, O.; Tyurikova, O.; Semyanov, A. Activity-dependent changes in transporter and potassium currents in hippocampal astrocytes. Brain Res. Bull. 2018, 136, 37-43. [CrossRef] [PubMed] 